\title{
Image Segmentation Algorithm Using Watershed Transform and Fuzzy C-Means Clustering on Level Set Method
}

\author{
Tara Saikumar, P. Yugander, P. S. Murthy, and B. Smitha, Member, IACSIT
}

\begin{abstract}
A new method for image segmentation is proposed in this paper, which combines the watershed transform, FCM and level set method. The watershed transform is first used to presegment the image so as to get the initial partition of it. Some useful information of the primitive regions and boundaries can be obtained. The fuzzy cmeans (FCM) was used to generate an initial contour curve which overcomes leaking at the boundary during the curve propagation. FCM algorithm computes the fuzzy membership values for each pixel. On the basis of FCM the edge indicator function was redefined. Using the edge indicator function of a MRI image was performed to extract the boundaries of objects on the basis of the presegmentation. Therefore, the proposed method is computationally efficient. Moreover, the algorithm can localize the boundary of the regions exactly due to the edges obtained by the watersheds. The efficiency and accuracy of the algorithm is demonstrated by the experiments on the MR brain images. The above process of segmentation showed a considerable improvement in the evolution of the level set function.
\end{abstract}

Index Terms-Image segmentation, watershed transform, level set method, FCM, MR brain image.

\section{INTRODUCTION}

Image segmentation is plays an important role in the field of image understanding, image analysis, pattern identification The foremost essential goal of the segmentation process is to partition an image into regions that are homogeneous (uniform) with respect to one or more self characteristics and features. Clustering has long been a popular approach to untested pattern recognition. Image segmentation is important in the field of image understanding, image analysis, pattern recognition and computer vision. The principal goal of the segmentation process is to partition an image into regions that are homogeneous with respect to one or more characteristics and features. Traditional segmentation algorithms are effective on the extraction of rigid objects. But, due to the impact of lighting in imaging process, sometimes the boundaries of object we get are not real, especially on the process of objects with varied topology structure, such as brain MR image; the traditional algorithms can't determine the real boundaries.

The level set method [1]-[6] is based on geometric deformable model, which translates the problem of evolution 2D (3D) close curve (surface) into the evolution of level set function in the space with higher dimension to obtain the

Manuscript received August 20, 2012; revised December 6, 2012.

Tara Saikumar is with CMR Technical Campus, Hyderabad, Andhra Pradesh (e-mail: tara.sai437@gmail.com) advantage in handling the topology changing of the shape. The level set method has had great success in computer graphics and vision. Also, it has been widely used in medical imaging for segmentation and shape recovery [7], [8]. However, there are some insufficiencies in traditional level set method. Firstly, as using the local marginal information of the image, it is difficult to obtain a perfect result when there's a fuzzy or discrete boundary in the region, and the leaking problem is unescapably appeared; Secondly, solving the partial differential equation of the level set function requires numerical processing at each point of the image domain which is a time consuming process; Finally, if the initial evolution contour is given at will, the iteration time would increase greatly, too large or too small contour will cause the convergence of evolution curve to the contour of object incorrectly. Therefore, some modification has been proposed to improve the speed function of curve evolution [9]-[11]. In the paper, based on the new variational level set method, the edge indicator function was weighted to improve the ability of detecting fuzzy boundaries of the object. At the same time, the FCM algorithm [12], [13] was applied to obtain the appropriate initial contour of evolution curve, so as to get the accurate contour of object and reduce the evolution time.

\section{WATERSHED ALGORTHIM}

In geography, a watershed is the ridge that divides areas drained by different river system. The watershed transform is a morphological gradientbased segmentation technique. The gradient map of the image is considered as a relief map in which different gradient values correspond to different heights. If we punch a hole in each local minimum and immerse the whole map in water, the water level will rise over the basins. When two different body of water meet, a dam is built between them. The progress continues until all the points in the map are immersed. Finally the whole image is segmented by the dams which are then called watersheds and the segmented regions are referred to as catchment basins. A catchment basin is the geographical area draining into a river or reservoir. The watershed algorithm applies these ideas to grayscale image processing in a way that can be used to solve a variety of image segmentation problem. Watershed algorithm, a segmentation method in mathematics morphology, was firstly introduced to the image division area by Beucher and Meyer.[14]-[19] It bases its concept on the restructure of measured lines in geodesy.[20], [21] In detail, it regards the image as the topological terrain in geodesy. In the image, the gray level value of every pixel stands for the 
altitude of a certain spot and different areas of gray level value correspond to different geological features. The calculating process with this algorithm can be likened a submerging process by a flood. Firstly, the flood submerges the lowest point in the image and gradually the whole valley. When the water level reaches a certain height, it will overflow at a certain place where the dam can be built. Repeat the process until all the spots in the image. At this moment, the series of completed dams will be the watershed separating every basin. Direct application of the watershed algorithm to a gradient image usually leads to over segmentation due to noise and other local irregularities of the gradient. The resulting problems can be serious enough to render the result virtually useless. A practical solution to this problem is to limit the number of allowable regions by incorporating a preprocessing stage designed to bring additional knowledge into the segmentation procedure [22]. An approach used to control over segmentation is based on the concept of controlled marker, which is proposed by Meyer and Beucher [19]. This approach is based on the idea that a machine vision system knows from other sources the location of the objects to be segmented.

\section{FUZZY CMEANS CLUSTERING (FCM)}

The standard fuzzy cmeans objective function for partitioning $\left\{x_{k}\right\}_{k=1}^{N}$ into $c$ clusters is given by

$$
J(U, V)=\sum_{i=1}^{c} \sum_{k=1}^{N} u_{i k}^{p}\left\|x_{k}-v_{i}\right\|^{2}
$$

where $\left\{x_{k}\right\}_{k=1}^{N}$ the feature vectors for each pixel are, $\left\{v_{i}\right\}_{i=1}^{c}$ are the prototypes of the clusters and the array $\left[u_{i k}\right]=U$ represents a partition matrix, namely

$$
\begin{aligned}
& \qquad \sum_{i=1}^{c} u_{i k}=1 \mid 0 \leq u_{i k} \leq 1, \forall k=1,2,3 \ldots . N \\
& \text { and } \quad 0 \leq \sum_{k=1}^{N} u_{i k} \leq N
\end{aligned}
$$

The parameter $p$ is a weighting exponent on each fuzzy membership and determines the amount of fuzziness of the resulting classification. The FCM objective function is minimized when the high membership values are assigned to pixels whose intensities are close to the centroid of their particular class, and low membership values are assigned when the pixel data is far from the centroid.

The constrained optimization could be solved using one Lagrange multiplier

$$
F_{m}=\sum_{i=1}^{c} \sum_{k=1}^{N} u_{i k}^{p}\left\|x_{k}-v_{i}\right\|^{2}+\lambda\left(1-\sum_{i=1}^{c} u_{i k}\right)
$$

where $\lambda$ denotes a Lagrange multiplier. The derivative of

$F_{m}$ w.r.t $u_{i k}$ was computed and the result was set to zero, for $p>1$

$$
\begin{gathered}
\frac{\partial F_{m}}{\partial u_{i k}}=p u_{i k}^{p-1}\left\|x_{k}-v_{i}\right\|^{2}-\lambda \\
u_{i k}=\left(\frac{\lambda}{m}\right)^{\frac{1}{m-1}} \frac{1}{\left\|x_{k}-v_{i}\right\|^{\frac{2}{m-1}}}
\end{gathered}
$$

The identity constraint $\sum_{j=1}^{c} u_{j k}=1 \forall k$ was taken into account,

$$
\left(\frac{\lambda}{m}\right)^{\frac{1}{m-1}} \sum_{j=1}^{c} \frac{1}{\left\|x_{k}-v_{j}\right\|^{\frac{2}{m-1}}}=1
$$

This allows us to determine the Lagrange multiplier $\lambda$

$$
\left(\frac{\lambda}{m}\right)^{\frac{1}{m-1}}=\frac{1}{\sum_{j=1}^{c} \frac{1}{\left\|x_{k}-v_{j}\right\| \frac{2}{m-1}}}
$$

The zerogradient condition for the membership estimator can be rewritten as

$$
u_{i k}=\frac{1}{\sum_{j=1}^{c}\left(\frac{\left\|x_{k}-v_{i}\right\|}{\left\|x_{k}-v_{j}\right\|}\right)^{\frac{2}{p-1}}}
$$

As no constraints, the computations of the prototypes were straightforward, The minimum of $J$ was computed with respect to $v_{i}$, and yielded the following equation

$$
\nabla_{v_{i}} J=0
$$

The detailed solution depends on the distance function.

In the case of the Euclidean distance, this leads to the expression:

$$
2 \sum_{k=1}^{N} u_{i k}^{p}\left(x_{k}-v_{i}\right)=0
$$

So the following could be immediately obtained

$$
v_{i}=\frac{\sum_{k=1}^{N} u_{i k}^{p} x_{k}}{\sum_{k=1}^{N} u_{i k}^{p}}
$$

The FCM algorithm for segmenting the image into different clusters can be summarized in the following steps:

FCM Algorithm:

Step 1: Select initial class prototype $\left\{v_{i}\right\}_{i=1}^{c}$.

Step 2: Update all memberships $u_{i k}$ with Eq. (9).

Step 3: Obtain the prototype of clusters in the forms of weighted average with Eq. (12). 
Step 4: Repeat step 23 till termination. The termination criterion is $\left\|V_{\text {new }}-V_{\text {old }}\right\| \leq \varepsilon$.

where $\|\cdot\|$ is the Euclidean norm. $V$ is the vector of cluster centers $\varepsilon$ is a small number that can be set by user (here $\varepsilon=0.01)$.

\section{The Modification to the LeVEl Set Method}

The level set method was invented by Osher and Sethian [3] to hold the topology changes of curves. A simple representation is that when a surface intersects with the zero plane to give the curve when this surface changes, and the curve changes according with the surface changes. The heart of the level set method is the implicit representation of the interface. To get an equation describing varying of the curve or the front with time, we started with the zero level set function at the front as follows:

$$
\phi(x, y, t)=0, \text { if }(x, y) \in 1
$$

Then computed its derivative which is also equal to zero

$$
\frac{\partial \phi}{\partial t}+\frac{\partial \phi}{\partial x} \cdot \frac{\partial x}{\partial t}+\frac{\partial \phi}{\partial y} \cdot \frac{\partial y}{\partial t}=0
$$

Converting the terms to the dot product form of the gradient vector and the $x$ and $y$ derivatives vector, we go

$$
\frac{\partial \varphi}{\partial t}+\left(\frac{\partial \varphi}{\partial x} \cdot \frac{\partial x}{\partial t}\right) \times\left(\frac{\partial \varphi}{\partial y} \cdot \frac{\partial y}{\partial t}\right)=0
$$

Multiplying and dividing by $\nabla \phi$ and taking the other part to be $F$ the equation was gotten as follows:

$$
\frac{\partial \phi}{\partial t}+F|\nabla \phi|=0
$$

According to literature [9]11], an energy function was defined:

$$
E(\phi)=\mu E_{\mathrm{int}}(\phi)+E_{\text {ext }}(\phi)
$$

where $E_{\text {ext }}(\phi)$ was called the external energy, and $E_{\text {int }}(\phi)$ was called the internal energy. These energy functions were represented as:

$$
\begin{gathered}
E_{\text {int }}(\phi)=\int_{\Omega} \frac{1}{2}(\nabla \phi-1)^{2} d x d y \\
E_{e x t}(\phi)=\lambda L_{g}(\phi)+v A_{g}(\phi) \\
L_{g}=\int_{\Omega} g \delta(\phi)|\nabla \phi| d x d y \\
A_{g}=\int_{\Omega} g H(-\phi) d x d y \\
g=\frac{1}{1+\left|\nabla G_{\sigma} * I\right|}
\end{gathered}
$$

where $L_{g}(\phi)$ was the length of zero level curve of $\phi$; and $A_{g}$ could be viewed as the weighted area; I was the image and $g$ was the edge indicator function. In conventional(traditional) level set methods, it is numerically necessary to keep the evolving level set function close to a signed distance function[14], [15]. Reinitialization, a technique for occasionally reinitializing the level set function to a signed distance function during the evolution, has been extensively used as a numerical remedy for maintaining stable curve evolution and ensuring desirable results.

From the practical viewpoints, the reinitialization process can be quite convoluted, expensive, and has subtle side effects [16]. In order to overcome the problem, Li et al [8] proposed a new variational level set formulation, which could be easily implemented by simple finite difference scheme, without the need of reinitialization. The details of the algorithm are in the literature [8]. However, because only the gradient information was imposed in the edge indicator function, Li's method has a little effect on the presence of fuzzy boundaries.

In the paper, a innovative method was proposed to modify the algorithm. The original image was partitioned into some sub images by FCM. The fuzzy boundary of each sub image was weighted by $\alpha$, the edge indicator function was redefined:

$$
g^{\prime}=g+\alpha \cdot g_{2}
$$

where $g_{2}=\frac{1}{1+\left|\nabla G_{\sigma} \times I_{1}\right|}$

$I_{1}$ Was the image after clustering. The iterative equation of level set functional was:

$$
\frac{\left(\varphi^{n+1}-\varphi^{n}\right)}{\tau}=\mu\left[\Delta \varphi-\operatorname{div}\left(\frac{\nabla \varphi}{|\nabla \varphi|}\right)\right]+\lambda \delta(\varphi) \operatorname{div}\left(g \frac{\nabla \varphi}{|\nabla \varphi|}\right)+\operatorname{vg} \delta(\varphi)
$$

Taking $g^{\prime}=g+\alpha \cdot g_{2}$ into 24

$$
\phi^{n+1}=\phi^{n}+\tau\left\{\begin{array}{l}
\mu\left[\nabla \phi-\operatorname{div}\left(\frac{\nabla \phi}{|\nabla \phi|}\right)\right] \lambda \delta(\phi) \operatorname{div}\left(g \frac{\nabla \phi}{|\nabla \phi|}\right) \\
+v g \delta(\phi)+\alpha\left[\begin{array}{l}
\left.\lambda \delta(\phi) \operatorname{div}\left(g_{2} \frac{\nabla \phi}{|\nabla \phi|}\right)+\right] \\
v g_{2}(\phi)
\end{array}\right\}
\end{array}\right\}
$$

where $\alpha \in[0,1]$. When processing images of weak boundary or low contrasts, a bigger $\alpha$ was taken; otherwise, a smaller $\alpha$ was taken.

\section{The Generation of Initial CONTOURCURVE}

On the basis of FCM clustering in brain MR image segmentation, the over segmentation usually exists. In this paper, the result of FCM was used as initial contour curve, and the automated initialization of deformation model was finished. For all the pixels in each cluster i.e. white matter, if 4 neighborhoods included the heterogeneous pixel, the pixel 
was regarded as candidate boundary point. So the algorithm of curve tracing [17] was proposed. The exterior boundary of the cluster was tracked in the candidate boundary points. Finally, the closed curve was obtained. The steps of image segmentation with adapted level set method were as follows:

Step1. Set the number of clusters, then the original image was processed with FCM, and calculate the $g_{2}$.

Step2. Choose one cluster, evaluate the inside area with $-\rho$ and the outside area with $+\rho, \rho$ is a plus constant. The boundary of the area is set to 0 . The region of interest is defined initial contour.

Step3. Minimize the overall energy functional with 25 formula.

\section{EXPERIMENTAL RESULTS}

The segmentation of image takes an important branch in the surgery navigation and tumor radiotherapy. In the experiment, The image data from the IBSR(Internet Brain Segmentation Repository) are included to test the accuracy and efficiency of the proposed algorithm. The output of watershed transformation algorithm Fig. 1 is as shown below. Firstly the original MRI brain image is as shown in Fig (a) is transformed to a proposed watershed algorithm is that a superimposed image of ridge lines and original binary image, note the over segmentation. The approximate contour of white matter was got by FCM algorithm shown in Figure h of Fig. 2. The snooping of regions else appear as a result of the in excess of segmentation.

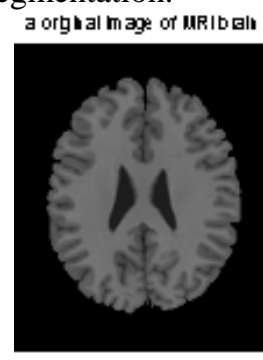

Figure a

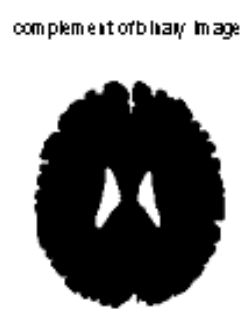

Figure d supe imposed mage

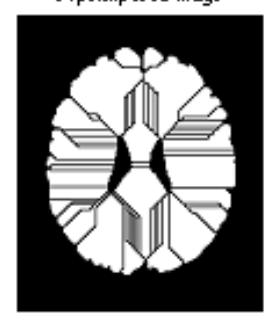

Figure $g$

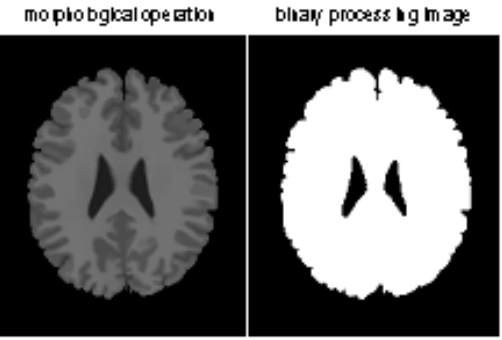

Figure c

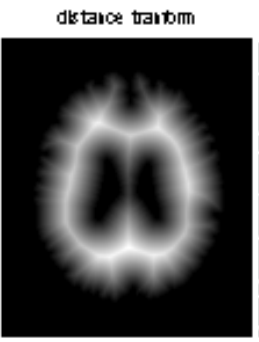

Figure e

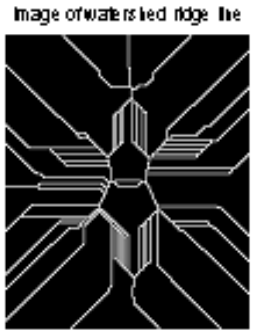

Figuref
Fig. a is the original test images (MRI Image)

Fig. $b$ is the morphological operation,

Fig. $\mathrm{c}$ is the binary processing image,

Fig. $d$ is the Complementary of binary image

Fig. e is the distance transform,

Fig. $f$ is the image of watershed ridge line,

Fig. $g$ is the superimposed image of ridge lines and original image.

Fig. $i$ are the results of final contour with proposed method.

$\begin{array}{lll}\text { Figure g } & \text { Figure h } & \text { Figure i } \\ \text { Superimposed } & \text { FCM } & \text { Proposed Final } \\ \text { Test Image } & & \text { contour, 300iteration }\end{array}$

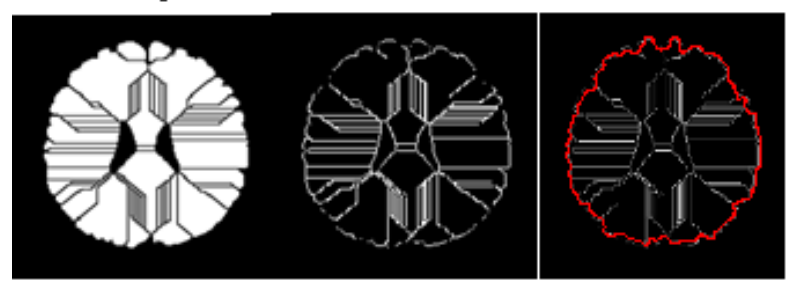

Fig. 2.

Fig. $h$ are the results of FCM clustering, to extracting the white matter.

With the enhanced method, the curve was successfully evolved to the hollow white matter boundaries, but only to the approximately white matter boundaries with Li's method. At the same time, because the curve has been converged to the narrow region the object boundaries extraction could not be implemented with Li's method. But the enhanced method solved this problem better. On the similar computing proposal, under a $3.0 \mathrm{GHz}$ Pentium iv PC with $1 \mathrm{~GB}$ RAM on board, the average processing time of improved method was $9.6 \mathrm{~s}$, and that was $30.3 \mathrm{~s}$ with Li's method. The evolution time was greatly reduced.

\section{DISCUSSIONS}

The need of the reinitialization is completely eliminated by the proposal of Chunming $\mathrm{Li}$, for pure partial differential equation driven level set methods, the variational level set methods. It can be easily implemented by using simple finite difference method and is computationally more efficient than the traditional level set methods. But, in this algorithm, the edge indicator has little effect on the low contrast image. Meanwhile, the initial contour of evolution needs to be determined by manual, and it has the shortcomings of timeconsuming and user intervention.

In this paper, we projected a new method to transform the algorithm. The original image was partitioned with FCM, and the controlled action of the edge indicator function was increased. The result of FCM segmentation was used to obtain the initial contour of level set method. With the new edge indicator function, results of image segmentation showed that the improved algorithm can exactly extract the corresponding region of interest.

\section{CONCLUSIONS}

In conclusion, the results of this study confirmed that the combination of FCM with the level set methods could be used 
for the segmentation of low contrast images, such as MR images. The method has the advantages of no reinitialization, automation, and reducing the number of iterations. The validity of new algorithm was verified in the process of exacting tissues of the brain. In the future research, the effect of priori information on the object boundary extraction with level set method, such as boundary, shape, and size, would be further analyzed. At the same time, the performance of image segmentation algorithms would be improved by reconstruction of classic velocity of level set method.

In the future research, noise was added in images prior information on the object boundary extraction with level set method, such as boundary, shape, and size, would be further analyzed. At the same time, the performance of image segmentation algorithms would be improved by modernization of classic velocity of level set method.

\section{REFERENCES}

[1] J. C. Bezdek, Pattern recognition with fuzzy objective function algorithms, Plenum Press, New York, 1981.

[2] K. L. Wu and M. S. Yang, "Alternative cmeans clustering algorthims," Pattern Recognition, vol. 35, pp. 2267-2278, 2002.

[3] S. Osher and J. A. Sethian, "Front propagating with curvature dependent speed: algorithms based on hamiltonjacobi formulations," Journal of Computational Physics, vol. 79, pp. 1249, 1988.

[4] R. Malladi, J. Sethain, and B. Vemuri, "Shape modelling with front propagation: A level set approach," IEEE Trans. Pattern Analysis and Machine. Intelligence, pp. 158174, 1995.

[5] L. Staib, X. Zeng, R. Schultz, and J. Duncan. "Shape constraints in deformable models," Handbook of Medical Imaging, I. Bankman (ed.), Academic Press, vol. 9, pp. 147-157, 2000.

[6] M. Leventon, O. Faugeraus, W. Grimson, and W. Wells, "Level set based segmentation with intensity and curvature priors," IEEE Workshop on Mathematical Methods in Biomedical Image Analysis Proceedings, pp. 411, 2000.

[7] N. Paragios and R. Deriche, "Geodesic active contours and level sets for the detection and tracking of moving objects," IEEE Transaction on pattern Analysis and Machine Intelligence, pp. 266280, Mar. 200.

[8] L. A. Vese and T. F. Chan, "A multiphase level set frame wor for image segmentation using the mumford and shah model," International Journal of Computer Vision, vol. 50, no. 3, pp. 271-293, 2002.

[9] Y. G. Shi and W. C. Karl, "Realtime tracking usin g level set," IEEE Computer Society Conference on Computer Vision and Pattern Recognition, pp. 34-42, 2005.

[10] C. Li, C. Xu, C. Gui, and M. D. Fox, "Level set evolution without reinitialization:a new varitional formulation," IEEE Computer Society Conference on Computer Vision and pattern Recognition, pp. 430-436, 2005.

[11] J. Sethain, Level set Methods and Fast Marching Methods, Cambridge University Press 1999.

[12] J. C. Dunn, "A fuzzy relative of the ISODATA process and its use in detecting compact wellseparated Clusters," Journal of Cybernetics, vol. 3, no. 3, pp. 3257, 1973.

[13] J. Bezedek, "A convergence theorem for the fuzzy ISODATA clustering algorthims," IEEE Trans. Pattern Analysis and Machine Intelligence, pp. 7882, 1980.

[14] S. Osher and R. Fedkiw, "Level set methods and dynamic implicit surfaces," Springer, vol. 57, no. 3, pp. 112-113, 2002.
[15] D. Peng, B. Merriman, S. Osher, H. Zhao, and M. Kang, "A PDE based fast local level set method," Journal of Computational Physics, vol 155, no. 2, pp. 410438, 1996.

[16] J. Gomes and O. Faugeras, "Reconciling distance functions and level Sets," J. Visual Communic and Image Representation, vol. 11, pp. 209223, 2000.

[17] T. McInerney and D. Terzopoulos, "Deformable models in medical image analysis: A survey," Medical Image Analysis, vol. 1, no. 2, pp. 91108, 1996.

[18] D. Q. Zhang and S. C. Chen, "Clustering in completed data usig Kernelbased fuzzy cmeans algorthim," Neural Processing Letters, vol. 18, no. 3, pp. 155162, December 2003.

[19] F. Meyer and S. beucher, "Morphology segmentation," Journal Visual Communications and Image Representation, vol. 1, no. 1, pp. 2126, 1990.

[20] J. Sijbers, M. Verhoye, P. Scheunders, D. V. Dyck, and E. Raman, "Watershed based segmentation of 3D MR data for volume quantization," Magnetic Resonance Imaging, vol. 15 no. 6 pp. 679-688, 1997.

[21] L. Vincent and P. Soille, "Watersheds in digital spaces: An efficient algorithmbased on immersion simulations," IEEE Transactions on PatternAnalysis and Machine Intelligence, vol. 13, no. 6, pp. 583-589, June 1991

[22] R. González and R. Woods, Digital image processing, Second Edition, Publishing House of Electronics Industry, pp. 505-507, 2003.

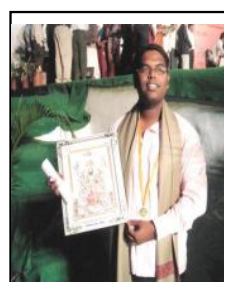

Tara. Saikumar was born in 1987 in Hyderabad, India. He received the B. Tech. degree in Electronics and Communication Engineering from Joginpally. B. R. Engineering College, Jawaharlal Nehru Technical University, Hyderabad in 2008. And the M. Tech. degree from Kakatiya Institute of Technology and Science (KITS), Warangal, India, in 2010 . He is the university topper in his M.Tech, KITS, Warangal and also received gold medal from Dr. D. N. Reddy vicechancellor JNTUH in his B.Tech for his excellence20042008. He has more than 35 papers in his credit, International journal, International and National Conferences. He is a life member of IACSIT, and UAEEE. He got Best paper award in 2011. He is currently a Asst Professor with the Dept of Electronics and Communication Engineering, CMR Technical Campus, Hyderabad. His research interests include digital image processing, digital signal processing, communication and neural network.

P. Yugander received his B. Tech., degree in Electronics \& Communication Engineering from Raja Mahendra College of Engineering, Hyderabad, M.Tech., degree in Digital Communications from Kakatiya Institute of Technology and Science, Kakatiya University, Warangal. Presently working as Assistant Professor in

ECE, KITS Warangal.

P. Sreenivasa Murthy obtained B. E., (CSE) from Osmania University, Hyderabad in 1995, M. E., (CSE) Sathyabama University, Chennai in 2006 and presently pursing Ph.D from Sant Gadge Baba Amaravati University, Amaravati. Presently working as Professor in the Dept. of Computer Science, CMR Institute of Technology, Hyderabad since Jul'2010. Life Member of ISTE, Member of International Association of Computer Science and Information Technology (IACSIT). Published a text book on Data Structures through $\mathrm{C}++$. Expert Committee member for CDAC, Hyderabad.

B. Smitha received his B. Tech., degree in Electronics \& Instrumentation Engineering from Vaagdevi College of Engineering, Warangal, M. Tech., degree in Digital Communications from Kakatiya Institute of Technology and Science, Kakatiya University, Warangal. Presently working as Assistant Professor in EIE, KITS Warangal. 